\title{
LOS PROGRAMAS INFORMÁTICOS DE SUBTITULACIÓN EN LA ENSEÑANZA/APRENDIZAJE DE LA TAV DENTRO DEL ÁMBITO UNIVERSITARIO (1): UNA PROPUESTA DE EVALUACIÓN Antonio Roales Ruir. \\ Universidad Complutense de Madrid
}

\section{ABSTRACT}

The increasing interest for Audiovisual Translation in high-level Translation Studies has shown the need of specific subtitling software. Most of the software used in a professional way is not suitable for teaching. The present paper studies the main problems that arise during the teaching process of Audiovisual Translation and sets a methodology of analysis of the subtitling software used in Translation Studies. The aim of the investigation is to determinate if this software is appropriate for teaching and learning how to create subtitles.

KEY WORDS: Audiovisual Translation, teaching and learning process, Subtitling, IT, Constrained Translation.

\section{RESUMEN}

El creciente interés por la TAV en las aulas universitarias y la inclusión en los planes de estudios de los estudios de Traducción e Interpretación en España de asignaturas dedicadas a esa disciplina - $-\mathrm{y}$, más concretamente, a la subtitulación- ha traído consigo el uso de software para la preparación de subtítulos. No obstante, estos programas no están exentos de problemas cuando se aplican a la didáctica. El presente artículo trata de analizar cuáles son esos problemas al mismo tiempo que explica una metodología de análisis de los programas informáticos de subtitulación que se suelen utilizar en nuestras aulas con el fin de detectar sin son adecuados para enseñar y aprender a subtitular.

PALABRAS CLAVE: Traducción Audiovisual, enseñanza-aprendizaje, subtitulación, TIC, traducción subordinada.

FECHA DE RECEPCIÓN: 19/04/2015

FECHA DE ACEPTACIÓN: 29/06/2015

PÁGINAS: 761-774 
1. INTRODUCCIÓN

Existe en el mercado una gran variedad de software específico para la subtitulación. Muchos de los programas son de gran calidad y permiten llevar a cabo con máximas garantías la labor para la que han sido pensados, pero su enfoque no es —ni tiene por qué serlo— didáctico.

En nuestra vertiente profesional de la subtitulación hemos tenido $-\mathrm{y}$ seguimos teniendo- ocasión de trabajar frecuentemente con varios de esos programas. Por otro lado, nuestra experiencia docente nos ha permitido analizar simultáneamente este tipo de software desde una perspectiva académica, para tratar de estudiar qué aspectos de los programas con los que habitualmente trabajan — trabajamos- los profesionales se ajustan a las necesidades de los estudiantes de TAV y también en qué medida permiten una adecuada parametrización que se adhiera a la diversidad de normas técnicas que pueblan el panorama de la teoría y la práctica de la subtitulación.

Nuestro convencimiento de que los problemas que plantea una disciplina tan específica como la subtitulación solo pueden resolverse en la medida en que los estudiantes cuenten con las herramientas adecuadas nos ha llevado en un primer momento a querer analizar los programas de subtitulación que hoy existen, porque creemos que resulta muy complicado utilizarlos con fines didácticos.

Algún programa, como es el caso de Subtitul@m, se propuso en su momento cubrir parte del vacío existente en la didáctica de la subtitulación; pero sus resultados, a nuestro entender, fueron poco satisfactorios, como ya tratamos de demostrar en un trabajo anterior (Roales 2008a). En sus conclusiones argumentábamos que con este programa:

[...] puede llevarse a cabo parte del trabajo que realiza el subtitulador y puede tenerse una idea aproximada de las diversas fases por las que transita el traductor que se enfrenta a la subtitulación de cualquier material fílmico; pero carece de bastantes funciones muy específicas —e indispensables - que deben mostrar al estudiante los pasos necesarios a la hora de enfrentarse a un material de estas características. Echamos en falta sobre todo aquellas que tienen que ver con aspectos «restrictivos» de este tipo de traducción. No podemos olvidar que estamos hablando de una de las especialidades dentro del ámbito de la traducción cuya esencia es precisamente la «restricción». La necesidad de respetar escrupulosamente parámetros que no tienen que ver con lo puramente lingüístico, sino con variables espaciales y temporales directamente ligadas a la imagen, es de la máxima importancia. 
La realidad parece haber demostrado que, pese a su loable finalidad, el programa no cumplía con los objetivos que se había planteado, puesto que a partir de junio de 2007 dejó de comercializarse.

En líneas generales, el software de subtitulación profesional está bien diseñado, es estable, potente y cumple con la práctica totalidad de requisitos que un profesional de la subtitulación necesita para llevar a cabo su labor. En definitiva, sirve para desempeñar la labor para la que ha sido creado: la preparación, edición y emisión de subtítulos profesionales; pero conviene apuntar determinadas características que hacen que, a priori, no podamos hablar de herramientas especialmente válidas para cumplir con la labor de enseñanza y aprendizaje:

1. Están pensados para mejorar la productividad de las empresas que los utilizan, pero no para formar a sus traductores. Se entiende que estos son profesionales formados que ya dominan su manejo o que tienen la suficiente base formativa como para aprender a utilizarlos con soltura en un corto espacio de tiempo.

2. Cuentan con infinidad de funciones avanzadas que a menudo no se emplean y complican en exceso los primeros pasos del aprendizaje. Ejercitarse para sacarles partido y para exprimir las funciones con las que cuentan suele requerir varias semanas de trabajo.

3. El uso de su interfaz general de trabajo, las barras de herramientas y los menús pueden resultar complicados en una primera fase.

4. En algunos casos cuentan con diversas versiones, específicas para cada canal de emisión (DVD, televisión, cine, emisión televisiva de subtítulos en directo, etc.), lo que hace difícil decantarse por una de ellas.

5. No es inusual tener que adquirir software adicional que permita, por ejemplo, la inclusión del código de tiempo en la pantalla.

6. Su elevadísimo precio hace prácticamente imposible la adquisición de varias licencias para su uso en la Universidad.

7. Si el precio de adquisición de una licencia resulta exorbitante para una institución educativa, para un estudiante la opción se vuelve absolutamente prohibitiva. De modo que, incluso aceptando que en la facultad se cuente con una batería de licencias que permita el trabajo en el centro, queda prácticamente descartada la opción de trabajo en casa. El entrenamiento - procedimiento clave en el aprendizaje de esta materia - queda, pues, relegado a aquellos momentos en que el estudiante no tenga clase y se dé la coincidencia de que esté libre el aula donde se encuentra instalado el software. 
Por supuesto, el hecho de que no se trate de programas concebidos específicamente para la docencia y el aprendizaje no significa que no puedan incluso deban - presentarse y analizarse con detenimiento en las aulas; pero entendemos que debe hacerse en una segunda fase, una vez que el estudiante tenga bien asimilados los fundamentos teóricos, prácticos y técnicos propios de la subtitulación. Estamos convencidos de que su aproximación a esos programas resultará entonces mucho más rápida y, sin duda, productiva.

Por todo lo visto, debemos concluir que para enseñar a subtitular es imprescindible poder contar con herramientas informáticas que respondan a las necesidades específicas del proceso de enseñanza-aprendizaje de la subtitulación. Para resultar realmente efectivas, estas deberán, en primer lugar, poner sobre aviso al estudiante de los errores que vaya cometiendo y, en segundo, guiarlo en las diversas etapas del proceso de aprendizaje. En definitiva, creemos que es importante contar con alguna herramienta que cumpla con una doble función: por un lado, la de ir orientando y afianzando los pasos del subtitulador novel hacia la asimilación de los conceptos, técnicas y requisitos específicos de la subtitulación y por otro lado, la de ir acercándolo paulatinamente al funcionamiento del software profesional. Se trata, pues, de construir el escalón previo: el que conduce a un puente que vaya adentrándolo progresivamente en un ámbito tan especializado y profesional como el que nos ocupa.

Veamos cómo analizar los programas con los que contamos para saber en qué medida se adecuan a nuestro propósito: enseñar y aprender a subtitular.

\section{FASES DEL ESTUDIO}

Nuestro estudio sobre el software de subtitulación se ha desarrollado en tres fases, que detallamos a continuación:

\subsection{PRIMERA FASE: ELECCIÓN DE PROGRAMAS}

En esta primera fase se procedió a hacer un barrido amplio para conocer y obtener una copia de los editores de subtitulación más importantes. En nuestra recopilación hicimos una división en función de un criterio de análisis que nos parecía clave: por un lado seleccionamos aquellos programas que pertenecen a grandes empresas del sector de la subtitulación. Se trata de programas que hemos dado en llamar «profesionales». En este grupo, queríamos estudiar los siguientes programas: EZTitles IV, FAB subtitler 7 
Professional Edition, Spot 5, Sub Machine 4, WinCAPS Qu4ntum 4, Cavena Tempo 3 y Softni Subtitler Suite 2.

Por otro lado, consideramos que era muy interesante realizar también una selección amplia de programas que se han popularizado en estos últimos años en la Red y que más bien se circunscriben al ámbito amateur. Son programas que suelen utilizar los denominados fansubbers, pero algunos de esos programas gozan hoy de cierta reputación y se han introducido en las aulas universitarias por su sencillez de manejo y, sobre todo, por un elemento crucial: su gratuidad. A este grupo de programas los hemos denominado «semiprofesionales». En esta categoría pretendíamos ocuparnos de los siguientes: Aegisub, AHD Subtitles Maker Professional, DivXLand Media Subtitler, Jubler Subtitle Editor, Open Subtitle Editor, SubMagic, Subtitle Edit (Nikse), Subtitle Processor, Subtitle WorkShop y VisualSubSync. Existen muchos otros programas de este tipo, pero no hemos considerado oportuno analizarlos porque son extremadamente simples, cumplen apenas con el requisito de permitir la inclusión de tiempos de entrada y salida y la escritura de los textos que conforman los subtítulos. No permiten establecer las más elementales posibilidades de configuración con vistas a una preparación de subtítulos mínimamente útil en el campo educativo o profesional.

\subsection{SEGUNDA FASE: ESTABLECIMIENTO DE LOS CRITERIOS DE ANÁLISIS}

En esta fase nos propusimos elaborar una lista de características que considerábamos que todo software de subtitulación debería tener; en concreto, queríamos centrarnos en aquellas particularidades que harían del software en cuestión un programa adecuado para el aprendizaje de la técnica de subtitulación. Para ello parecía lógico apoyarse, entre otras cuestiones, en preceptos técnicos ampliamente aceptados por el ámbito académico y comprobar si estaban presentes o no (y en qué medida) en el abanico de programas estudiados. En concreto, decidimos verificar tales criterios en función de las siguientes preguntas:

1. ¿Dispone el software de onda de audio?

2. ¿Permite el software determinar la velocidad de lectura máxima?

3. En caso afirmativo, ¿̇en qué formato?

4. ¿Permite el software determinar el número máximo de caracteres por subtítulo?

5. En caso afirmativo, ¿muestra el programa alguna alerta si se supera el límite? 
6. ¿Permite el software determinar el número máximo de caracteres por línea?

7. En caso afirmativo, ¿muestra el programa alguna alerta si se supera el límite?

8. ¿Permite el software establecer algún tipo de margen de desvío sobre el número máximo de caracteres que puede admitir un subtítulo?

9. ¿Permite el software determinar la duración máxima de un subtítulo?

10. En caso afirmativo, ¿muestra el programa alguna alerta si se supera el límite?

11. ¿Permite el software determinar la duración mínima de un subtítulo?

12. En caso afirmativo, ¿muestra el programa alguna alerta si no se alcanza ese valor?

13. ¿Permite el software determinar el intervalo mínimo que debe existir entre dos subtítulos consecutivos?

14. En caso afirmativo, ¿muestra el programa alguna alerta si no se respeta ese límite?

15. ¿Avisa de alguna manera el software si el pautado de entrada de un subtítulo es anterior al de salida del precedente?

16. ¿Dispone el software de algún sistema que detecte los cambios de plano?

Como puede observarse, excepto la pregunta n. 3 (que completa la anterior), todas las demás resultan fácilmente objetivables. El planteamiento de la objetivación nació de la idea de que resultaría útil para nuestro propósito establecer algún sistema de puntuación que permitiera a posteriori una interpretación afinada y lo más imparcial posible de los datos obtenidos. Nuestra intención era, pues, tratar de realizar un análisis de parámetros que pudieran medirse de alguna manera.

Por otro lado, en nuestro análisis y posterior evaluación quisimos incluir un parámetro que considerábamos esencial en nuestro estudio: el precio. Por adaptado a la enseñanza de la subtitulación que pudiera resultar cualquier editor, si su coste no es asumible ni por los centros universitarios ni por los propios estudiantes, parece evidente que su valor como herramienta didáctica si no desaparece, al menos disminuye por una cuestión palmaria: la imposibilidad de acceder a él por lo desmesurado del coste. La objetivación de este dato no es particularmente compleja: disponer de una cifra permite la aplicación de criterios matemáticos.

Quedaba únicamente el establecimiento de un parámetro importante en nuestro estudio, cuya medición resultaba algo más compleja por la dificultad de objetivar el dato: la facilidad de uso. 
Marques (2000), realiza una acertada distinción entre los medios didácticos y los recursos educativos:

Teniendo en cuenta que cualquier material puede utilizarse, en determinadas circunstancias, como recurso para facilitar procesos de enseñanza y aprendizaje [...], pero considerando que no todos los materiales que se utilizan en educación han sido creados con una intencionalidad didáctica, distinguimos los conceptos de medio didáctico y recurso educativo.

Medio didáctico es cualquier material elaborado con la intención de facilitar los procesos de enseñanza y aprendizaje. Por ejemplo un libro de texto o un programa multimedia que permite hacer prácticas de formulación química.

Recurso educativo es cualquier material que, en un contexto educativo determinado, sea utilizado con una finalidad didáctica o para facilitar el desarrollo de las actividades formativas. Los recursos educativos que se pueden utilizar en una situación de enseñanza y aprendizaje pueden ser o no medios didácticos. Un vídeo para aprender qué son los volcanes y su dinámica será un material didáctico (pretende enseñar), en cambio un vídeo con un reportaje del National Geographic sobre los volcanes del mundo a pesar de que pueda utilizarse como recurso educativo, no es en sí mismo un material didáctico (sólo pretende informar).

Entre las funciones habituales que pueden desempeñar tanto los medios como los recursos educativos, el autor (ibid.) destaca las de proporcionar información, guiar los aprendizajes de los estudiantes (instruir); ejercitar habilidades (entrenar); motivar, despertar y mantener el interés; evaluar los conocimientos y las habilidades que se tienen; proporcionar simulaciones que ofrecen entornos para la observación, exploración y la experimentación, y proporcionar entornos para la expresión y creación.

Para el investigador (ibid.) evaluar significa:

[...] estimar en qué medida el elemento evaluado tiene unas características que se consideran deseables y que han sido especificadas a partir de la consideración de unos criterios. Por lo tanto toda evaluación exige una observación, una medición y un juicio.

En nuestro caso, los dos primeros parámetros evaluados (los requisitos técnicos y el precio) pueden ser observados y medidos. No así el tercero (la facilidad de uso), que puede ser observado, pero no medido (no al menos con 
los mismos criterios que los otros dos). Por ello, para la facilidad de uso tendremos que optar por la observación y por el juicio.

Profundizando en el aspecto de la evaluación, el autor antes citado (ibid.) realiza otras consideraciones que nos parecen relevantes:

[...] siempre que se realiza una evaluación hay una intencionalidad y unos destinatarios, la evaluación se hace para algo y para alguien, a partir de ella muchas veces se tomarán decisiones. Así, y centrándonos en la evaluación de medios didácticos, cuando se evalúan unos materiales se puede hacer para saber cuáles tienen más información sobre un tema, cuáles son los mejores desde un punto de vista técnico, cuáles son los más adecuados para unos estudiantes determinados, etc. Y por otra parte los destinatarios de esta evaluación pueden ser los docentes, los diseñadores de materiales didácticos, los administradores de las instituciones educativas...

En cualquier caso, los criterios que se utilicen deben estar de acuerdo con la intencionalidad de la evaluación y con los destinatarios de la misma.

Por otra parte, cuando consideramos la evaluación de los medios didácticos, uno de los criterios que siempre suele estar presente es el de la eficacia didáctica, es decir, su funcionalidad como medio facilitador de aprendizajes.

Para la evaluación de la citada eficacia didáctica el investigador (ibíd.) propone el método de la evaluación objetiva, que se centra en valorar la calidad de los medios didácticos. Y expone:

[...] generalmente la realiza un especialista a partir de un estudio exhaustivo de las características del material, sin que intervengan los destinatarios finales del medio didáctico.

En cualquier caso, la evaluación suele hacerse a partir de la consideración de unos criterios de calidad que se concretan en unos indicadores que se pueden identificar en mayor o menor medida en los materiales que se evalúan.

Los resultados de la evaluación se suelen recoger en unas plantillas "ad hoc" (más o menos extensas según el objeto y destinatarios de la evaluación) que incluyen diversos apartados: identificación del producto, valoración de acuerdo con los indicadores, evaluación global y comentarios.

Hemos optado, pues, por la elaboración de una ficha para nuestro estudio, que mostramos al final del presente apartado. Aunque existen varios modelos de ficha de catalogación y evaluación multimedia, hemos preferido 
elaborar una ad hoc atendiendo a la especificidad del ámbito que estamos estudiando: el análisis de la idoneidad del software de subtitulación profesional en el ámbito de la didáctica. En ella, hemos incluido tanto los datos que podemos observar y medir, como aquellos que solo podemos observar y juggar.

Los primeros (criterios técnicos y precio) pueden puntuarse directamente de forma numérica. Para el cómputo de las respuestas sobre los criterios técnicos, decidimos manejar un patrón binario, con el que solo se evalúa la presencia o ausencia del criterio. Así, asignamos a cada respuesta afirmativa un 1 y a cada respuesta negativa un $0^{1}$. Quince de las dieciséis preguntas efectuadas — todas salvo la tercera (formato en que se mide la velocidad de lectura en caso de que el software contemple esa posibilidad) — son, como ya hemos tenido ocasión de argumentar, fácilmente objetivables, pues a todas ellas puede responderse afirmativa o negativamente, sin necesidad de matizaciones. Se entiende, naturalmente, que una respuesta afirmativa en cada una de las preguntas técnicas realizadas en nuestro estudio supone un elemento deseable - y por tanto positivo- en un software de subtitulación. Al contrario, una respuesta negativa supone una carencia.

Para la valoración del parámetro precio, en cambio, sí tiene sentido establecer una escala numérica, puesto que existen diferencias claras de coste entre los distintos programas analizados. Puntuamos con un 0 el software más caro de todo el arco analizado y con un 10 aquel (o aquellos) más baratos (en la práctica, todos los gratuitos). Esto nos permitía promediar y obtener una transposición de puntuación de los programas de pago, empleando la técnica de interpolación de datos.

Por último, la evaluación de la facilidad de uso debía realizarse, como ya hemos argumentado, en función de criterios que exigían un juicio valorativo crítico por nuestra parte. Para ello, se elaboró una escala multinivel de apreciación según los criterios de valoración siguientes:

${ }^{1}$ No consideramos oportuno utilizar otro tipo de escala (del 1 al 10 por ejemplo) porque en este caso no hay posibilidad de gradación en la respuesta: el criterio evaluado existe o no existe. 


\begin{tabular}{|l|c|}
\hline \multicolumn{1}{|c|}{ Valor cualitativo } & Valor cuantitativo \\
\hline EXCELENTE & 10 \\
\hline ALTA & 7,5 \\
\hline CORRECTA & 5 \\
\hline BAJA & 2,5 \\
\hline NULA & 0 \\
\hline
\end{tabular}

Tabla 1: Escala multinivel para la valoración del criterio facilidad de uso

Ahora bien, pese al hecho de juzgar en función de nuestro criterio - lo que sin duda impregna de cierto grado de subjetividad el análisis de este punto concreto- hemos querido acotar los aspectos en función de los cuales hemos realizado nuestra valoración del parámetro. Para ello hemos recurrido a una interpretación de la norma ISO/IEC 9126 (2001), que clasifica la calidad del software en un conjunto estructurado de características y subcaracterísticas. Entre estas encontramos la de usabilidad, que podríamos definir como la capacidad del software para permitir que el usuario lo entienda, lo aprenda, lo use y lo valore cuando lo utiliza bajo determinadas condiciones. Las características de usabilidad responden a aspectos como:

La facilidad de comprensión: en qué medida el software permite al usuario entender los conceptos que el propio software encierra y en qué medida le aclara si es apropiado para sus necesidades.

La facilidad de aprendizaje: capacidad del programa para reducir el esfuerzo necesario por parte del usuario en el aprendizaje.

La operatividad: capacidad que proporciona el programa para que los usuarios lo utilicen por sus propios medios y controlen su uso.

El atractivo: capacidad del software para resultar «amigable».

La conformidad: capacidad del programa para ajustarse a las normas o convenciones relativas a la usabilidad.

La usabilidad es, pues, fácilmente identificable con lo que nosotros entendemos por facilidad de uso, y por ello nuestro análisis y juicio en este terreno se ha apoyado en los aspectos que acabamos de detallar como criterio valorativo. 
Si tenemos en cuenta la puntuación por criterios técnicos, le añadimos el parámetro evaluador del precio e incorporamos la apreciación sobre la facilidad de uso, el rango de valoración abarca de los 0 a los 35 puntos (15 puntos máximos por parámetros técnicos +10 máximos por gratuidad del software +10 máximo por óptima facilidad de uso). Según la horquilla que acabamos de mencionar, un software sería tanto mejor cuanto más se aproximara a la cifra de 35 puntos. Cierto, pero creemos conveniente resaltar que la interpretación de resultados no debería hacerse según el criterio de media aritmética simple. Es decir, no creemos que deba recurrirse a una exégesis simplista según la cual aquellos programas que hayan obtenido una puntuación de 17,5 puntos puedan considerarse programas aptos para la didáctica de la subtitulación. En nuestra opinión, para una fiabilidad depurada de la valoración, los resultados de los tres criterios deben analizarse y valorarse previamente por separado y a continuación en conjunto, y parece razonable pensar que para que un programa sea apto (no decimos óptimo, sencillamente apto) debería alcanzar la media de puntuación en los tres criterios, puesto que los tres evalúan propiedades muy dispares, que no pueden combinarse. Poco valor didáctico tendría, a nuestro entender, un editor de subtitulación que fuera gratuito (10 puntos) y muy fácil de utilizar (7,5 puntos), pero que no contemplara en su configuración ni un parámetro técnico propio de nuestra disciplina. Tampoco resultaría adecuado aquel software que cumpliendo con todos los requisitos técnicos (15 puntos) y mostrando una usabilidad correcta (5 puntos), tuviera un coste de 3000 euros ni siquiera 300 parecen abordables_-, sencillamente porque sería inviable que tanto el profesor como todos los estudiantes lo poseyeran. Como vemos, para una correcta evaluación enfocada a nuestro propósito, es necesaria una doble valoración: para que un software pueda considerarse apto, debería alcanzar una puntuación mínima de 17,5 puntos, pero al mismo tiempo esa suma tendrá que provenir de valores que indefectiblemente habrán de superar la media en los tres apartados que forman parte de nuestros criterios de análisis. A saber:

1. Criterios técnicos: 7,5 puntos

2. Precio: 5 puntos

3. Facilidad de uso: 5 puntos

Como puede observarse, si se mantiene ese equilibro de puntuación, la suma alcanza igualmente los 17,5 puntos mínimos, pero de manera armónica y compensada. A partir de estos supuestos, ahora sí, creemos que puede considerarse que cualquiera de los editores analizados será tanto mejor cuanto más se aproxime a la cifra de 35 puntos, que obviamente sería la óptima. 
Presentamos a continuación la ficha de evaluación del software que hemos elaborado para el análisis de los distintos editores, y que recoge todos los elementos de valoración previamente expuestos:

\section{FICHA DE ANÁLISIS DE SOFTWARE}

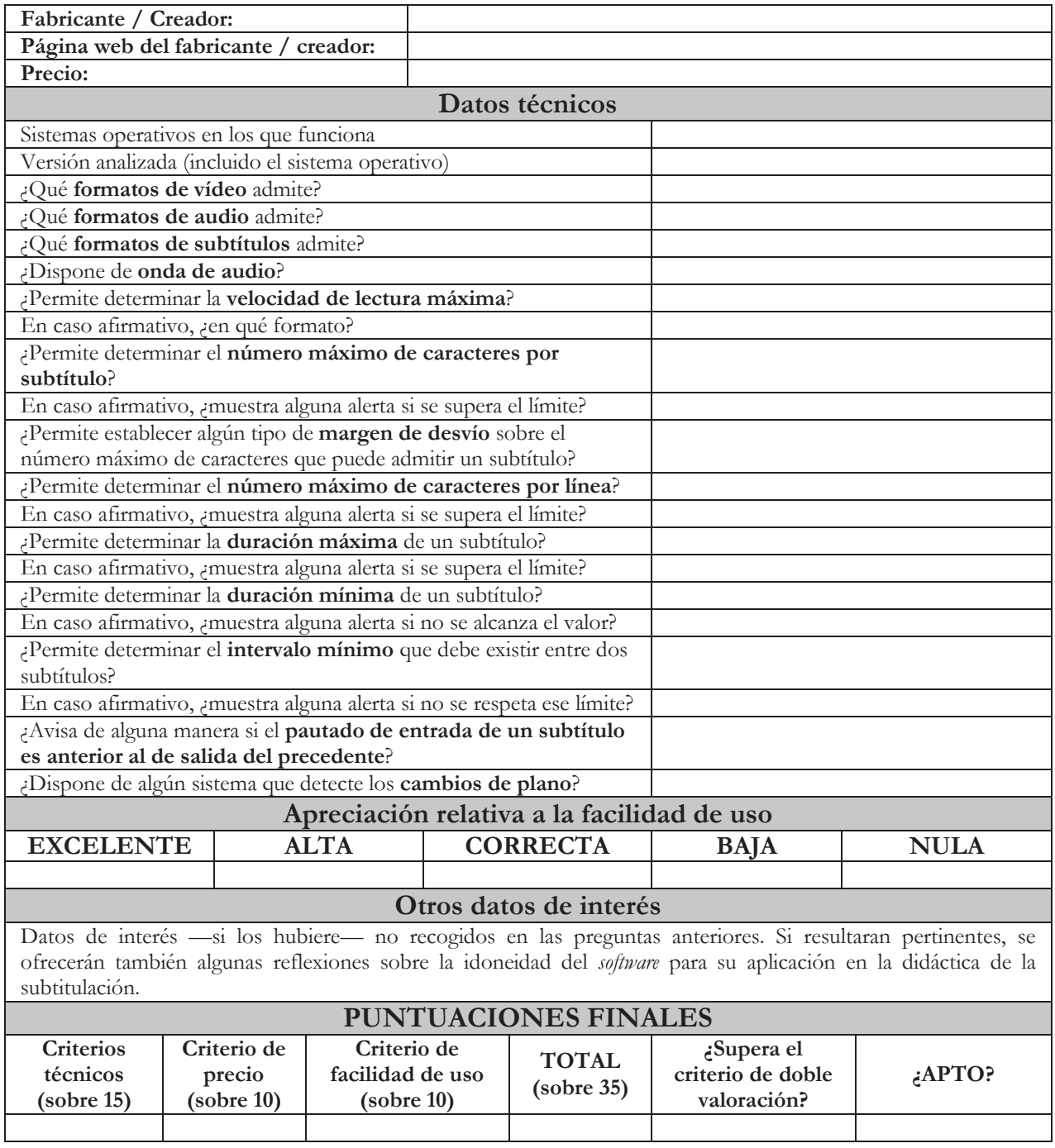

\subsection{TERCERA FASE: ANÁLISIS DEL SOFTWARE}

En la tercera fase, se procedió a realizar la instalación de todos los programas y a comprobar por el método de prueba directa si cumplían con los requisitos técnicos que habíamos establecido en la fase anterior. Para ello, 
procedimos a seleccionar una pequeña secuencia de unos dos minutos de duración y a efectuar el proceso de subtitulación completo con todos los programas analizados. Primeramente, tratamos de fijar en la sección oportuna de cada programa los parámetros técnicos que consideramos esenciales para el desarrollo de una buena subtitulación, entendiendo — como ya hemos tenido ocasión de comprobar - que estos parámetros son variables; pero buscábamos que fuera posible, al menos, establecerlos; es decir, queríamos saber hasta qué punto el software analizado permitía ajustarse a las reglas técnicas comúnmente aceptadas tanto en el ámbito académico como por la profesión. Verificamos también si los criterios técnicos establecidos tenían alguna traducción en sistemas de aviso claros y precisos para el subtitulador mientras elaboraba su trabajo.

En una segunda etapa, nos dedicamos a consignar en la ficha aquellos datos no relacionados con los criterios técnicos de la subtitulación. Queríamos conocer algunos datos de índole más informativa que propiamente técnica (formatos de audio, vídeo y subtítulos admitidos por el programa, el fabricante, su página web, el precio, etc.). Para ello consultamos la documentación técnica, los manuales de usuario, la propia página web del producto o incluso, en varias ocasiones, recurrimos a la pregunta directa al fabricante sobre datos como el precio por licencia del producto.

Por último, una vez probado en profundidad, procedimos a evaluar los aspectos relativos a la usabilidad según los criterios que hemos detallado anteriormente.

Hasta aquí el planteamiento del problema y la metodología propuesta para su estudio. La pormenorización de los resultados, así como las conclusiones del análisis de todos los programas estudiados se presentarán en artículos posteriores dada la magnitud y especificidad del trabajo.

\section{BIBLIOGRAFÍA}

Cerezo, B. (2012). La didáctica de la traducción audiovisual en España: Un estudio de caso empirico-descriptivo. Tesis doctoral. Castellón, Departamento de Traducción y Comunicación de la Universidad Jaume I.

Díaz Cintas, J. (2001). La traducción audiovisual. El subtitulado. Salamanca, Almar. Díaz Cintas, J. (2003). Teoría y práctica de la subtitulación (inglés-español). Barcelona, Ariel.

Gómez Pérez, M. y Roales Ruiz, A. (2011). «Uso didáctico de la subtitulación en la clase de Lengua $\mathrm{C} \gg$ en Roiss, S. et al. (eds.). En las vertientes de la Traducción e Interpretación del/ al Alemán. Berlín, Franklin \& Timme, pp. 201212. 
González-Iglesias, J. D. (2012). Desarrollo de una berramienta de análisis de los parámetros técnicos de los subtítulos y estudio diacrónico de series estadounidenses de televisión en DVD. Tesis doctoral. Salamanca, Departamento de Traducción e Interpretación de la Universidad de Salamanca.

Hurtado, A. (1999). «Objetivos de aprendizaje y metodología en la formación de traductores e intérpretes», en Hurtado, A. (ed.). Enseñar a traducir. Metodología en la formación de traductores e intérpretes. Madrid, Edelsa, pp. 8-58.

ISO (International Organization for Standardization) (2001). Software Product Evaluation. Quality Characteristics and Guidelines for their Use. ISO/IEC Std 9126, ISO.

Ivarsson J. y Carroll M. (1998). Subtitling. Simrishamn: TransEdit HB.

Karamitroglou, F. (1998). «A proposed set of subtitling standards in Europe», en Translation Journal, vol. 2, núm. 2. Disponible en: http://translationjournal.net/journal/04stndrd.htm.

Lachat, C. (2011). «Didáctica de la traducción audiovisual: enseñar a mirar», en Redit 6, pp. 94-105.

Marques, P. (2000). Los medios didácticos. Disponible en: http://peremarques.pangea.org/medios2.htm\#inicio.

Mayoral, R. (1998). «Aspectos curriculares de la enseñanza de la traducción e interpretación en España», en García, I. y Verdegal, J. (eds.). Los estudios de traducción: un reto didáctico. Castellón de la Plana, Publicacions de la Universitat Jaume I, pp. 117-130.

Roales, A. (2008a). «Estudio crítico de una aplicación informática para la didáctica de la subtitulación: Subtitul@m»en Enlaces: revista del CES Felipe II - UCM, núm. 9.

Disponible en: http://www.cesfelipesegundo.com/revista/numeros.html.

Roales, A. (2008b). Trabajo de DEA. Salamanca, Departamento de Traducción e Interpretación de la Universidad de Salamanca.

Roales, A. (2014). Estudio crítico de los programas de subtitulación profesionales. Carencias en su aplicación para la didáctica. Propuesta de solución mediante conjunto de aplicaciones integradas. Tesis doctoral. Salamanca, Departamento de Traducción e Interpretación de la Universidad de Salamanca.

Toda, F. (2008). «Teaching Audiovisual Translation in a European Contex๖», en, J. Díaz Cintas (ed.) (2008), pp. 157-168.

Torres del Rey, J. (2003). Nuevas tecnologías y enseñanza de la traducción: limites y posibilidades de los modelos de aplicación tecnológica para la formación de traductores. Tesis doctoral. Universidad de Salamanca, Departamento de Traducción e Interpretación. 